\title{
Epigenetic studies in Developmental Origins of Health and Disease: pitfalls and key considerations for study design and interpretation
}

\author{
L. Yamada and S. Chong* \\ Epigenetics Group, Translational Research Institute, Mater Research Institute - The University of Queensland, Woolloongabba, QLD, Australia
}

\begin{abstract}
The field of Developmental Origins of Health and Disease (DOHaD) seeks to understand the relationships between early-life environmental exposures and long-term health and disease. Until recently, the molecular mechanisms underlying these phenomena were poorly understood; however, epigenetics has been proposed to bridge the gap between the environment and phenotype. Epigenetics involves the study of heritable changes in gene expression, which occur without changes to the underlying DNA sequence. Different types of epigenetic modifications include DNA methylation, post-translational histone modifications and non-coding RNAs. Increasingly, changes to the epigenome have been associated with early-life exposures in both humans and animal models, offering both an explanation for how the environment may programme long-term health, as well as molecular changes that could be developed as biomarkers of exposure and/or future disease. As such, epigenetic studies in $\mathrm{DOHaD}$ hold much promise; however, there are a number of factors which should be considered when designing and interpreting such studies. These include the impact of the genome on the epigenome, the tissue-specificity of epigenetic marks, the stability (or lack thereof) of epigenetic changes over time and the importance of associating epigenetic changes with changes in transcription or translation to demonstrate functional consequences. In this review, we discuss each of these key concepts and provide practical strategies to mitigate some common pitfalls with the aim of providing a useful guide for future epigenetic studies in $\mathrm{DOHaD}$.
\end{abstract}

Received 22 April 2016; Revised 11 July 2016; Accepted 10 August 2016; First published online 9 September 2016

Key words: DNA methylation, environmental exposures, histone modifications, non-coding RNAs, study design

\section{Introduction}

Early-life environmental exposures are thought to influence organ development and physiology such that there is an increased risk of disease in later life. ${ }^{1,2}$ Growing evidence suggests that early-life exposures can also impact the epigenome. Epigenetics has been defined in a number of ways ${ }^{3-5}$ and the field has yet to reach a generally accepted consensus. For the purposes of this review, we define epigenetics as the study of heritable changes in gene expression, which occur without changes to the underlying DNA sequence. ${ }^{6}$ Epigenetic marks have the capacity to be stably inherited through successive mitotic cell divisions, providing a possible molecular 'memory' of the exposure, and can be associated with altered gene expression, thereby affecting phenotype. As such, epigenetics has the potential to both further our understanding of the mechanisms which underlie the link between early-life exposures and later health outcomes, and to produce novel molecular biomarkers of past exposure and/or future disease. ${ }^{7}$ Here, we cover essential concepts for epigenetic studies in Developmental Origins of Health and Disease (DOHaD),

*Address for correspondence: Dr S. Chong, Epigenetics Group, Mater Research Institute - The University of Queensland, Translational Research Institute, Level 4, 37 Kent Street, Woolloongabba, QLD 4102, Australia. (Email Suyinn.chong@mater.uq.edu.au) identify potential hazards in study design and interpretation, and highlight strategies for the generation of informative and meaningful results.

\section{Epigenetics}

\section{DNA methylation}

Within mammals, cytosine methylation is the most wellcharacterized DNA modification. Cytosine methylation occurs most frequently within cytosine phosphate guanine $(\mathrm{CpG})$ dinucleotides, with $70-80 \%$ of $\mathrm{CpGs}$ within the human genome methylated. ${ }^{8}$ Non-CpG cytosine methylation also occurs but is tissue-specific, with higher levels reported in oocytes, ${ }^{9}$ pluripotent cells ${ }^{10}$ and various regions of the brain. ${ }^{11-13}$

Cytosine methylation is catalyzed by the highly conserved DNA methyltransferase (DNMT) family of proteins. ${ }^{14-16}$ DNMT1 has a higher affinity for hemi-methylated than unmethylated DNA and is responsible for propagating methylation after DNA replication, thus acting to maintain methylation states. ${ }^{14}$ DNMT3A and DNMT3B are essential for the establishment of new, or de novo methylation marks. ${ }^{15}$ Although DNMT3L lacks catalytic activity, ${ }^{17}$ it can bind to and stimulate the catalytic activity of DNMT3A and DNMT3B. ${ }^{18,19}$ 
Until recently, little was known about how DNA was demethylated. Several mechanisms had been proposed for the active demethylation of DNA, including those involving DNA deamination by methyl-CpG-binding domain protein 4 (MBD4) and glycosylation ${ }^{20,21}$ and cytosine deamination by DNMTs. ${ }^{22,23}$ There is a growing body of literature supporting another mechanism mediated by the Ten-Eleven Translocation family of proteins, which sequentially hydroxylate 5-methylcytosine to 5-hydroxymethylcytosine, 5-formylcytosine and finally to 5-carboxylcytosine. ${ }^{24-26}$ Through thymine DNA glycosylation followed by base excision repair, 5-carboxylcytosine is then converted back to the unmodified cytosine. ${ }^{25}$

Passive, replication-dependent DNA demethylation can also occur. ${ }^{27}$ In the early preimplantation mouse embryo, it was observed that chromosome methylation was iteratively lost with each cycle of DNA replication. ${ }^{28}$ This followed an earlier observation that DNMT1 was excluded from the cell nucleus in the very early stages of embryogenesis following fertilization, and only observable in the nucleus from the eight-cell stage. ${ }^{29,30}$ The absence of a maintenance DNMT from the nucleus would result in a passive reduction in global methylation state with every cell division.

CpG-rich regions, called CpG islands, are often found at the 5 promoter region of genes, ${ }^{31}$ and methylation of these regions is associated with transcriptional silencing. ${ }^{32,33}$ Promoter methylation is believed to prevent transcriptional initiation. ${ }^{34}$ In contrast, intragenic $\mathrm{CpG}$ methylation in mammalian cells has little effect on transcriptional initiation, instead discouraging transcriptional elongation. ${ }^{35}$ Intragenic methylation can also aid in exon recognition, playing a role in the regulation of alternative splicing. ${ }^{36,37}$

DNA methylation appears to be responsive to the environment, with alterations in DNA methylation patterns reported in both humans and animals following a range of adverse earlylife exposures, including those of malnutrition, alcohol, choline and arsenic. ${ }^{38-44}$ The $\mathrm{DOHaD}$ field has historically focused on promoter DNA methylation; however, the use of unbiased genome-wide screens for DNA methylation has identified associations between early-life exposures and methylation of non-promoter regions such as enhancers ${ }^{45}$ as well as intergenic regions. ${ }^{46,47}$ Mechanistically, there is some evidence that early-life exposures to alcohol and choline deficiency in rodents can alter the expression of the maintenance methyltransferase Dnmt $1 .{ }^{41,48,49}$ One-carbon metabolism is a network of pathways involved in a number of functions, including the synthesis of methionine which can subsequently be adenosylated to $S$-adenosyl methionine - a major source of methyl groups necessary for DNA methylation. ${ }^{50}$ One-carbon metabolism may also mediate environmentally induced changes to DNA methylation as it can be perturbed by early-life exposures to alcohol ${ }^{51}$ and maternal smoking ${ }^{52}$ as well as by alterations in gestational maternal intake of methyl donors such as choline and folate. ${ }^{48,53-55}$ Disrupting either Dnmt1 levels or onecarbon metabolism would be expected to impact DNA methylation genome-wide. Although global alterations to DNA methylation have been reported following certain exposures, ${ }^{55}$ many exposures fail to induce such changes, instead resulting in locus-specific effects. ${ }^{45,48,56}$ How perturbations to Dnmt1 expression or one-carbon metabolism could induce locus-specific methylation changes remains unclear, and further study is required to understand the mechanisms by which the environment influences the methylome.

\section{Post-translational histone modifications}

In the nucleus, DNA is packaged into chromatin, the individual building blocks of which are nucleosomes. Within the nucleosome, DNA is wrapped around a protein octamer, comprising of two each of histone $\mathrm{H} 2 \mathrm{~A}$, histone $\mathrm{H} 2 \mathrm{~B}$, histone $\mathrm{H} 3$ and histone $\mathrm{H} 4 .^{57}$ Although the C-terminal domains of histones are critical for the maintenance of nucleosome structure, ${ }^{57}$ the $\mathrm{N}$-terminal tails function to alter the accessibility of the associated DNA. ${ }^{58}$ The $\mathrm{N}$-terminal tail of any histone can, at specific amino acid positions, undergo chemical modifications including acetylation, methylation, ${ }^{59}$ phosphorylation, ${ }^{60}$ ubiquitinylation, ${ }^{61}$ carbonylation, ${ }^{62}$ poly (ADP-ribosyl)ation ${ }^{63}$ or sumoylation. ${ }^{64}$ These modifications are thought to alter chromatin structure by affecting electrostatic interactions between the DNA and histones, making them either more, or less tightly packaged and permissive of transcription. In addition, modified histones can be recognized by and directly interact with various proteins which can further modify the histones and/or affect chromatin structure. ${ }^{65}$ For example, the recognition of histone $\mathrm{H} 3$ lysine 4 trimethylation (H3K4me3) by inhibitor of growth 2 results in the recruitment and stabilization of the mSin3a-histone deacetylase 1 (HDAC1) complex at the gene promoter. ${ }^{66}$ In contrast, SWItch/sucrose non-fermentable is a chromatin remodeling complex which recognizes acetylated histones. ${ }^{67}$

The histone code hypothesis posits that the combinatorial identity and position of each $\mathrm{N}$-terminal tail modification acts as a code, controlling transcription in a highly specific manner. ${ }^{68}$ Individually, $\mathrm{H} 3 \mathrm{~K} 4 \mathrm{me} 3$ at gene promoters is associated with transcriptional activation, ${ }^{69}$ whereas trimethylation of lysine 27 at histone $\mathrm{H} 3(\mathrm{H} 3 \mathrm{~K} 27 \mathrm{me} 3)$ is associated with transcriptional repression. ${ }^{70}$ However, bivalent domains that have both activating (H3K4me3) and repressive (H3K27me3) marks simultaneously also exist. ${ }^{71}$ Occurring near gene promoters, bivalent domains are thought to poise genes for expression. ${ }^{71}$

Though epigenetic studies in $\mathrm{DOHaD}$ have primarily focused on DNA methylation, post-translational histone modifications are also subject to the influence of the early-life environment. ${ }^{41,72-74}$ The mechanism by which this occurs; however, is yet to be fully understood. Gestational choline deficiency has been identified to alter the expression of genes involved in the conferral of histone modifications, including the histone lysine methyltransferase Set domain bifurcated 1 (Setdb1) and histone methyltransferase G9a (Kmt1c). ${ }^{41,75}$ One-carbon metabolism may also be involved as $S$-adenosyl 
methionine, which in addition to being required for DNA methylation, also contributes to the post-translational methylation of histone tails. ${ }^{50}$ Given that early-life exposures can influence one-carbon metabolism, ${ }^{48,51-55}$ there is potential for these exposures to have consequences on histone as well as DNA methylation but, again, widespread changes might be expected. In support of this, global changes to histone methylation have been reported in rodents following various early-life exposures, including those of gestational choline deficiency ${ }^{76}$ and nicotine exposure. ${ }^{77}$ In contrast, locus-specific effects were observed when H3K4me3 was assayed by chromatin immunoprecipitation and next generation sequencing in the dentate gyrus of inbred C57BL/6 mice following an early-life exposure to arsenic. ${ }^{78}$ Further, a maternal high-fat diet produced coding region-specific changes in histone $\mathrm{H} 3$ lysine 9 trimethylation at the rat Wingless-type MMTV integration site family member 1 $(W n t 1)$ gene in offspring liver, ${ }^{79}$ suggesting the presence of mechanisms which allow for conferral of locus- and regionspecificity. Therefore, while it is evident that changes in post-translational histone modifications are associated with early-life exposures, further study is required to elucidate both how this occurs and how it impacts offspring health.

\section{Non-coding RNAs}

Non-coding RNAs can also affect gene expression, either by transcriptional or post-transcriptional mechanisms. Long noncoding RNAs influence gene expression using a wide array of mechanisms. ${ }^{80}$ For example, the Antisense Igf $2 r$ RNA (Air) long non-coding RNA accumulates at the Slc22a3 promoter and recruits the histone $\mathrm{H} 3 \mathrm{~K} 9$ methyltransferase G9a protein, thereby inducing locus-specific transcriptional repression in the placenta. ${ }^{81}$ For a more detailed discussion on the various mechanisms by which long non-coding RNAs influence transcription, we direct the reader to the review by Wang et al. ${ }^{80}$

Small non-coding RNAs can interact with nascent transcripts as well as with single- and double-stranded DNA in a sequence-specific manner. ${ }^{82}$ The major categories of small non-coding RNAs include endogenous short interfering RNAs (which are presently poorly characterized in mammals ${ }^{83}$ ), P-element induced wimpy testis-interacting RNAs (PIWIinteracting RNAs or piRNAs; expressed primarily in germ cells ${ }^{84}$ ), and microRNAs (miRNAs) (relatively well characterized and expressed in many tissues $\left.{ }^{85}\right) .{ }^{86}$ Of these small non-coding RNAs, miRNAs have been the subject of particular interest within the $\mathrm{DOHaD}$ field and as such, the remainder of this discussion will focus on miRNAs.

miRNAs are -22 nucleotides in length, and bind to the 3 ' untranslated region (UTR) of target messenger RNAs (mRNA) in order to post-transcriptionally regulate their stability and/or translation into protein. ${ }^{87}$ miRNAs regulate target mRNA levels by cleavage ${ }^{88}$ or degradation. ${ }^{89}$ Although some studies have reported exclusive effects of miRNAs on protein translation, ${ }^{90,91}$ others have reported instances whereby miRNAs first inhibit protein translation, and are then subsequently involved in mRNA deadenylation and decay. ${ }^{92}$ The extent of base-pair complementarity between the miRNA and target mRNA can influence whether a given miRNAs inhibits or aids translation. ${ }^{93}$ Further, Let-7 and a synthetic miRNA (miRcxcr4) were identified to upregulate translation at certain points during the cell cycle, but at other times, the same miRNAs repressed translation of the same target reporter construct. ${ }^{94}$

miRNAs were previously estimated to regulate between 20 and $30 \%$ of human genes. ${ }^{95,96}$ However, since the initial estimates were generated, a large number of new miRNAs have been discovered, rendering the figures relatively conservative. Each miRNA has been estimated to target 100-200 mRNAs, with miRNAs likely to act coordinately to aid in the regulation of any given target gene. ${ }^{97,98}$

An increasing number of studies within the $\mathrm{DOHaD}$ field are reporting changes in the expression of both long noncoding $\mathrm{RNAs}^{42}$ and miRNAs ${ }^{99-101}$ following an early-life exposure. A number of studies have also begun to identify circulating miRNAs, in plasma and serum, as potential biomarkers of various early-life exposures. ${ }^{101-104}$ Interest in non-coding RNAs in the context of $\mathrm{DOHaD}$ is relatively recent, and consequently little is known about either the mechanisms by which they are regulated or the downstream functional consequences.

\section{Interactions between epigenetic modalities}

In $\mathrm{DOHaD}$ studies, the epigenetic modalities of DNA methylation, histone modifications and non-coding RNAs are often considered in isolation, but there is substantial evidence that they regulate gene expression in concert with each other.

\section{DNA methylation and histone modifications}

The co-dependent nature of DNA methylation and histone modifications was nicely demonstrated when Zhang et al. ${ }^{105}$ observed that in order to achieve complete demethylation and activity of the luteinizing hormone receptor promoter in vitro, the addition of both a histone deacetylase inhibitor (trichostatin A) and a DNA demethylating reagent (5-azacytidine) were required. These results built on earlier findings in which the binding of methyl-CpG-binding protein 2 (MeCP2) to methylated DNA was shown to recruit histone deacetylases to impact locally on histone acetylation and chromatin structure. ${ }^{106-108}$

Likewise, both histone modifications themselves as well as the proteins responsible for conferring histone modifications can impact DNA methylation. For example, protein arginine methyltransferase 5 (PRMT5) confers symmetric methylation of arginine 3 at histone $\mathrm{H} 4$ (H4R3me2s) which then acts as a binding target for DNMT3A. ${ }^{109}$ Oocytes deficient in a H3K4 demethylase (KDM1B) exhibited genome-wide DNA hypomethylation, suggesting a critical role of $\mathrm{H} 3 \mathrm{~K} 4$ demethylation in DNA methylation regulation; however, the exact mechanism for this has not yet been elucidated. ${ }^{110}$ The histone methyltransferase Enhancer of Zeste homolog 2 (EZH2) 
directly recruits DNMTs, ${ }^{111}$ and DNMT3L can bind to histone $\mathrm{H} 3$ when its lysine 4 is unmethylated, inducing de novo DNA methylation by DNMT3A2. ${ }^{112}$

For further information on the complex interplay between DNA methylation and histone modifications, we direct the reader to several reviews. ${ }^{13-115}$

\section{DNA methylation and non-coding RNAs}

DNA methylation can regulate the expression of non-coding RNAs including miRNAs. ${ }^{116}$ In turn, DNA methylation itself can also be influenced by various types of non-coding RNAs. DNA methylation can be directed in a sequence-specific manner through the direct interaction of DNMTs with long non-coding RNAs, including Tsix (the antisense transcript of Xist) and numerous promoter-associated non-coding RNAs. ${ }^{17-121}$ The imprinted $H 19$ non-coding RNA also indirectly regulates the activity of DNMT3B by binding to $S$-adenosylhomocysteine hydrylase, thereby interfering with the hydrolysis of $S$-adenyosylhomocysteine - an inhibitor of DNMT3B. ${ }^{122}$

Short non-coding RNAs can also influence DNA methylation. When MitoPLD, a protein involved in primary piRNA synthesis was mutated in mice, the de novo DNA methylation of the RAS protein-specific guanine nucleotide-releasing factor 1 (RasgrfI) differentially methylated region (DMR) in spermatogonia was impaired, suggesting a role for piRNAs in de novo DNA methylation. ${ }^{123}$ Similarly, the PIWI proteins MILI and MIWI2, which interact with piRNAs, were essential for the establishment of de novo methylation of retrotransposons in male fetal germ cells. ${ }^{124} \mathrm{~A}$ number of miRNAs, which include miR-148a and miR-152, are able to directly target the expression of Dnmt1. ${ }^{125,126}$

\section{Histone modifications and non-coding RNAs}

Although histone modifications can regulate the expression of non-coding $\mathrm{RNAs}^{127,128}$, non-coding RNAs themselves are also capable of directing histone modifications. ${ }^{129,130}$ The long non-coding RNA, HOTAIR, facilitates the conferral of histone modifications to the Homeobox D cluster by acting as a scaffold for both the polycomb repressive complex 2 (PRC2) and the Lysine-specific demethylase 1 (LSD1)/coRepressor element-1 silencing transcription factor (coREST)/RE1-silencing transcription factor (REST) complex, which in turn recruit enzymes to trimethylate histone $\mathrm{H} 3$ lysine 27 and demethylate histone $\mathrm{H} 3$ lysine 4, respectively. ${ }^{131,132}$ A number of histone modifying enzymes, including $\mathrm{HDAC} 1{ }^{133}$ and EZH $2,{ }^{134}$ have also been identified to be direct targets of miRNAs.

For further information regarding the interaction between non-coding RNAs and the other epigenetic modalities, we direct the reader to other reviews. ${ }^{129,130}$

\section{Epigenetic reprogramming in mammals}

There are two major developmental periods, preimplantation development and gametogenesis, when the epigenome is erased and reset genome-wide in a process called epigenetic reprogramming. ${ }^{135-138}$ It has been proposed that the epigenome is most susceptible to environmental exposures during these periods of epigenetic reprogramming. ${ }^{39}$

In preimplantation development, DNA methylation changes include an initial global demethylation event postfertilization, ${ }^{139}$ in which paternally derived DNA undergoes active demethylation, ${ }^{140}$ whereas maternally derived DNA undergoes replication-dependent passive demethylation. ${ }^{28}$ Methylation is then re-established de novo from implantation onwards (approximately gestational day 4.5 in the mouse), with somatic tissues becoming increasingly methylated. ${ }^{141}$ This reprogramming of the epigenome in the preimplantation embryo is necessary to allow cells of the early embryo to achieve a state of pluripotency, ${ }^{142}$ and to then set up distinct patterns of gene expression that are associated with differentiation and cell fate determination.

Imprinted genes, which are resistant to preimplantation epigenetic reprogramming, have been the subject of a number of studies in the $\mathrm{DOHaD}$ field. ${ }^{139}$ Although most genes are expressed from both the maternally and paternally derived alleles (biallelic expression), imprinted genes are expressed monoallelically - that is, exclusively from either the maternally or paternally derived allele. ${ }^{139}$ Imprinting is a known epigenetic process; many of these genes have well-characterized DMRs that are associated with and thought to control monoallelic expression. The finding that imprinted gene DMR methylation in somatic tissues can be altered by gestational environmental exposures ${ }^{143,144}$ appears at odds with their resistance to preimplantation epigenetic reprogramming; however, it is possible that the exposure compromises this resistance. Interestingly, other studies have identified no changes in imprinted gene DMR methylation in response to gestational environmental perturbations, despite identifying changes in expression, ${ }^{145,146}$ leading to speculation that these expression changes are due to transcription-factor-mediated mechanisms rather than epigenetic mechanisms. ${ }^{146}$ Indeed, the idea that imprinted regions are of no greater importance than any other genomic region in the epigenetic response to early-life exposures has been extensively discussed in a recent review. ${ }^{147}$

Later in development, a second major epigenetic reprogramming event occurs during gametogenesis. ${ }^{139}$ This reprogramming event involves the removal of parent-of-origin DNA methylation from imprinted loci, allowing for the establishment of new sex-specific methylation patterns, such that the alleles are imprinted with either a maternal pattern (in oocytes) or a paternal pattern (in sperm). This erasure of parental imprints begins during the migration of primordial germ cells to the genital ridge (from approximately gestational day 9.5 to 11.5 in the mouse). ${ }^{148}$ In murine male germ cells, DNA remethylation occurs when these primordial germ cells become prospermatogonia (from approximately gestational day 13 in the mouse) and is completed by birth. ${ }^{149}$ In contrast, DNA remethylation in female germ cells does not commence until after birth, occurring during preovulatory oocyte growth 
and maturation. ${ }^{135,150}$ Given that the timing of DNA remethylation is sexually dimorphic in nature, sensitivity to environmental exposures may also differ between sperm and oocytes. Furthermore, gametogenesis may be a period during which imprinted genes may be most sensitive to exposures. Specifically, differences in imprinting may be most likely to occur in offspring derived from males exposed during late gestation or offspring derived from females exposed in the preconceptional period (during oocyte maturation).

Given the difficulty of obtaining germ cell and/or preimplantation embryo samples from humans, much of the knowledge pertaining to epigenetic reprogramming has been obtained using murine models. Nonetheless, a small number of studies utilizing human samples have found that many of these processes appear to be conserved between species. ${ }^{151,152}$

Mammalian epigenetic reprogramming is a complex phenomenon, and while a very brief overview is provided here, we direct the reader to more comprehensive reviews for further information. ${ }^{135-138}$

\section{Considerations for epigenetic studies in $\mathrm{DOHaD}$}

As much promise as epigenetics has in unraveling the molecular mechanisms underlying adverse health outcomes following early-life environmental exposures, there are a number of challenges when conducting these studies. In this section, we discuss the central challenges in epigenetic studies in both humans and animal models, how they may impact the interpretation of results and highlight strategies to mitigate some of these issues (summarized in Tables 1 and 2).

\section{The epigenome is influenced by the genome}

In addition to the extrinsic influence of the environment, the epigenetic landscape is also shaped intrinsically by the underlying DNA sequence. For example, the comparison of adolescent and middle-aged monozygotic and dizygotic twins suggested a greater contribution of genetics than environmental factors to DNA methylation at the imprinted Insulin-like growth factor 2 (IGF2) DMR. ${ }^{153}$ Genetic influences on DNA methylation have also been reported genome-wide. When two well-characterized inbred mouse strains, C57BL/6 and BALB/c, with several hundred differentially methylated loci were mated, the F1 hybrid (C57BL/6xBALB/c) offspring exhibited strain-specific methylation patterns on each allele, likely driven by the local genomic context in $c i{ }^{154}$

In humans, widespread associations between single nucleotide polymorphisms and DNA methylation have been reported in methylation quantitative trait loci studies. ${ }^{155-157}$ The underlying mechanisms as to how these genetic variants influence methylation remain poorly understood; however it has been proposed that the creation or disruption of $\mathrm{CpG}$ sites, ${ }^{155}$ or perturbations in transcription factor binding ${ }^{156}$ may be involved. Another way in which the genome can influence the epigenome is via functional mutations within genes which contribute to the establishment or maintenance of epigenetic marks. For example, the 5, 10-methylenetetrahydrofolate reductase (MTHFR) gene encodes an enzyme critical for the supply of methyl donors for reactions such as DNA methylation. The C677T polymorphism within the human MTHFR gene results in reduced MTHFR activity ${ }^{158}$ and is associated with genomic DNA hypomethylation in peripheral leucocytes. ${ }^{159,160}$ Similarly, the R271Q polymorphism within the DNMT3L gene in humans is another example of a polymorphism resulting in DNA hypomethylation. ${ }^{161}$ There is limited literature to suggest that histone modifications too may be dependent on the local genomic context. ${ }^{162}$ Consequently, in $\mathrm{DOHaD}$ studies utilizing genetically heterogeneous populations such as humans or outbred animals, it can be difficult to distinguish between epigenetic changes driven by the environment and those driven by genetic differences, unrelated to the environment.

In both humans and outbred animals, genetic differences can be accounted for, if not controlled for, by

Table 1. Strategies to address common challenges in epigenetic studies

\begin{tabular}{ll}
\hline Challenges & Possible solutions \\
\hline Genetic heterogeneity & - Use inbred strains of animals \\
- Identify genetic polymorphisms: & o Identify known single nucleotide polymorphisms using online databases \\
& o Genotyping samples \\
& o Whole-genome sequencing \\
Functional consequences & - Assay messenger RNA and protein levels as well as phenotype \\
Cell- and tissue-specificity & - Assay epigenetic modifications in multiple tissues to determine tissue-specificity or lack thereof \\
& - Purify and assay single types of cells \\
& - Define the cellular composition of the tissue to be assayed
\end{tabular}


Table 2. A selection of recent DOHaD publications that have addressed two or more of the challenges highlighted in this review. Black boxes denote the challenge addressed by the study

\begin{tabular}{|c|c|c|c|c|c|c|c|}
\hline First author (year) & Species (strain) & Early-life exposure & Epigenetic modification & 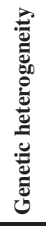 & 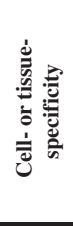 & 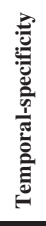 & 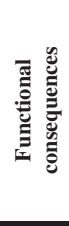 \\
\hline Novakovic (2014) & Human & Smoking & DNA methylation & & & & \\
\hline Breton (2014) & Human & Tobacco smoke & DNA methylation & & & & \\
\hline Kundakovic (2013) & Mouse (BALB/c) & Bisphenol A & DNA methylation & & & & \\
\hline Silver (2015) & Human & Peri-conceptional environment & DNA methylation & & & & \\
\hline Mehedint (2009) & Mouse (C57BL/6) & Choline deficiency & DNA methylation, histone modifications & & & & \\
\hline Zhang (2015) & Mouse (C57BL/6J) & Ethanol & DNA methylation, histone modifications, ncRNAs & & & & \\
\hline El Hajj (2013) & Human & Gestational diabetes mellitus & DNA methylation & & & & \\
\hline Amarasekera (2014) & Human & Folate & DNA methylation, histone modifications & & & & \\
\hline Kile (2014) & Human & Arsenic & DNA methylation & & & & \\
\hline Downing (2011) & Mouse (C57BL/6) & Ethanol/methyl-supplementation & DNA methylation & & & & \\
\hline Laufer (2013) & Mouse (C57BL/6J) & Ethanol & DNA methylation, ncRNAs & & & & \\
\hline Basil (2014) & Mouse (C57BL/6N) & Immune activation & DNA methylation & & & & \\
\hline Thompson (2010) & Rat (Sprague-Dawley) & Intrauterine growth restriction & DNA methylation & & & & \\
\hline Begum (2013) & Sheep (Romney) & Undernutrition & DNA methylation, histone modifications & & & & \\
\hline
\end{tabular}

assaying genotype. ${ }^{162-165}$ In rodents, genetic differences can be minimized through the use of inbred strains. A recent study identified few genetic or epigenetic differences of gross magnitude between C57BL/6 littermates. ${ }^{166}$ However, even this approach is not without problems. Using inbred mice, Shea et al. ${ }^{167}$ recently identified variation in DNA methylation at ribosomal DNA repeats in C57BL/6 sperm, which seemingly correlated with paternal diet. Upon further analysis, the difference in methylation was found to be an artifact of copy number variation at this repetitive element, and unrelated to paternal diet. ${ }^{167}$ Taken together, the evidence supports the importance of considering the contribution of genetic variation to epigenetic variation, even in inbred animals.

\section{Epigenetic modifications are cell- and tissue-specific}

Although each individual tends to have minimal genetic variation across tissues, the epigenome is reflective of the different and often dynamic transcriptional identities of tissues, and even individual cells. As such, any given individual, despite having only one genome, can have numerous epigenomes. ${ }^{12,168-173}$ When the methylomes of six regions from the brain as well as that of whole blood were compared in nine human donors by immunoprecipitation of methylated DNA followed by next generation sequencing, greater differences were observed between tissues within an individual than between the same tissue across individuals. ${ }^{171}$ Even within tissues, cell-to-cell variation in DNA methylation has been reported. ${ }^{174,175}$

Similarly, when epigenetic changes are identified in one tissue following an early-life exposure, comparable changes are not assured in other tissues. Maternal smoking during pregnancy was associated with hypomethylation of the Aryl hydrocarbon receptor repressor (AHRR) gene in newborn cord blood mononuclear cells, but not in buccal epithelium or placental tissue. ${ }^{176}$ This example of a tissue-specific response is not an isolated case, with methylation of long interspersed element 1 (LINE1) repeats, measured as a proxy for global DNA methylation, hypomethylated in the hypothalamus but not the striatum in offspring of a mouse model of prenatal maternal immune activation. ${ }^{177}$ Furthermore, Kundakovic et al. ${ }^{178}$ observed an increase in DNA methylation within the estrogen receptor 1 (EsrI) gene in the prefrontal cortex, but not hypothalamus of male offspring from an inbred $\mathrm{BALB} / \mathrm{c}$ mouse model of prenatal bisphenol A exposure. In the offspring from an ovine model of maternal undernutrition, despite the differential methylation of the glucocorticoid receptor gene in various regions of the brain, no evidence of differential methylation of this gene was identified in leucocytes. ${ }^{179}$ Therefore, caution is recommended particularly when inferring the epigenetic state of a disease-relevant, but inaccessible tissue based on the epigenetic state of another more accessible tissue.

The relatively accessible nature of whole blood makes it a commonly assayed tissue in many human studies of DNA methylation following an early-life environmental exposure. $^{56,180,181}$ There is however, a growing body of literature reporting considerable epigenetic heterogeneity within whole blood, reflective of its diverse cellular composition. ${ }^{182,183}$ When cellular composition was corrected for in silico in five published studies examining age-related DNA methylation changes, cellular composition explained a greater proportion of the reported epigenetic variation in these studies than did age. ${ }^{184}$ Subsequently, Bauer et al. ${ }^{185}$ found that a 
previously reported association between tobacco smoking and DNA methylation at the G protein-coupled receptor 15 (GPR15) locus was in fact an artifact of increased numbers of CD3+ $\mathrm{T}$-cells in the smoking population. Therefore, any study of epigenetic changes in whole blood or white blood cells should consider the cellular composition of these samples.

There are a number of approaches available to reduce the confounding influence of cellular heterogeneity. One solution is to sort samples into individual cell types; however, this will significantly reduce the amount of tissue available for analysis. As an alternative to this, a number of computational methods have been developed to estimate and account for differences in cellular distributions in heterogeneous tissues such as blood and brain, utilizing previously defined DNA methylation signatures for each cell type. ${ }^{186-190}$ These methods have been applied in some studies examining DNA methylation following early-life exposures. ${ }^{43,44,181}$ Future DOHaD studies will undoubtedly continue to assay blood and other heterogeneous tissues; however, the consideration of cellular heterogeneity should aid the meaningful interpretation of any epigenetic changes identified.

\section{Epigenetic modifications are not necessarily stable over time}

In addition to being influenced by genetics and cellular identity, the epigenome may also change with time. Global DNA methylation in the livers of male C57BL/6 mice was found to gradually decline from the ages of 6-24 months, ${ }^{191}$ suggesting that aging can alter methylation profiles. A similar study of DNA methylation differences in lymphocytes between monozygotic twins ranging in age from 3 to 74 found that while younger twins had relatively few epigenetic differences, the magnitude of difference within twin pairs increased with age across multiple tissues. ${ }^{192}$ In this study, the contribution of genetic heterogeneity to epigenetic variation was reduced through the utilization of monozygotic twins. ${ }^{192}$ Similar trends in methylation differences were also observed in four separate tissue types, suggesting that the impact of cellular composition on the epigenetic profiles was minimal. ${ }^{192}$ It is possible, even likely, that in addition to aging, the observed methylation changes reflect differences in postnatal exposures between twins over life. Regardless of the underlying causes, these studies draw attention to the idea that DNA methylation patterns can change over time.

The stability of epigenetic modifications over time also appears to be gene-dependent. When methylation of DNA isolated from saliva samples from adolescent monozygotic twin pairs were assayed before and after a period of several months, Levesque et al. ${ }^{193}$ found that even in this relatively short period of time, the methylation of 46 genes was unstable, whereas 226 genes were identified to be temporally stable. Another study reported similar outcomes, with DNA methylation at five of eight candidate loci studied longitudinally over 11-20 years deemed stable in both whole blood and buccal cells. ${ }^{194}$ In this study, the authors were able to account for genetic heterogeneity by assaying for sequence variation, and for cellular heterogeneity using computational methods. ${ }^{194}$

Most $\mathrm{DOHaD}$ studies assay for epigenetic changes in samples collected at a single time-point, ${ }^{38,40-43,45-47}$ often far removed from the environmental exposure itself. The evidence presented above highlights the importance of assaying for epigenetic changes on multiple occasions, and demonstrating stability over time, especially if the epigenetic marks are proposed to confer a memory of the exposure or to serve as biomarkers.

\section{The relationship between epigenetics and gene expression can be ambiguous}

Although DNA methylation patterns are often linked with transcriptional activity, this is not always the case. Indeed, in eight human tissues, 5'UTR DNA methylation status was inversely correlated with transcription for only $37 \%$ of the 43 genes analyzed. ${ }^{195}$ Similarly, a paternal low-protein diet was associated with considerable changes in both mRNA and miRNA expression as well as DNA methylation in offspring livers; however, the genes at which promoter methylation was altered were not necessarily those which displayed differential expression. ${ }^{196}$ In a separate study, the differential methylation of 181 gene promoters in pancreatic islets from patients with type 2 diabetes and healthy controls correlated with altered transcription for only $18 \%$ of the genes. ${ }^{197}$ It must be acknowledged that an epigenetic effect on adjacent locus expression at a different time-point cannot be excluded, nor an effect on the expression of other, more distantly located, genomic loci. In these instances however, such outcomes would need to be demonstrated experimentally.

A disconnect between transcriptional activity and DNA methylation has been observed at imprinted loci as well. The differential expression, but not DNA methylation, of a number of imprinted genes was identified in the livers of offspring exposed to gestational protein restriction. ${ }^{145}$ Further, when altered expression, but not methylation of the imprinted paternally expressed 3 (Peg3) gene was observed in a mouse model of maternal undernutrition, the authors speculated that this was likely due to transcription factor-mediated mechanisms, rather than epigenetic mechanisms per se. ${ }^{146}$ It is however possible that other epigenetic marks, such as post-translational histone modifications, may still be contributing to these outcomes, even in the absence of changes to DNA methylation.

The finding that mRNA and protein levels are not always positively correlated, ${ }^{101,198,199}$ suggests that protein analyses may also be informative in epigenetic studies in $\mathrm{DOHaD}$. The lack of correlation between mRNA and protein levels can potentially be explained by an epigenetic mechanism: miRNAs. In a model of gestational nutrient restriction, 23 miRNAs associated with the insulin-signaling pathway were identified to be differentially expressed in the liver of fetal lambs. ${ }^{200}$ The expression of these miRNAs were then found to correlate with target protein but not 
mRNA levels, ${ }^{200}$ suggesting that the miRNAs act on target protein translation rather than mRNA stability.

Our recent study which assayed DNA methylation, histone modifications and miRNA expression, as well as both mRNA and protein levels illustrates the informative potential of broader experimental designs. In this study, hippocampal tissue was assayed from adult male C57BL/6J mice following an early gestational ethanol exposure. ${ }^{101}$ Both a reduction in DNA methylation and increase in $\mathrm{H} 3 \mathrm{~K} 4 \mathrm{me} 3$ (a marker of active chromatin) were observed at the promoter region of a vesicular glutamate transporter gene, Slc17a6, in ethanol-exposed mice. As would be predicted, these epigenetic changes correlated with an increase in Slc17a6 mRNA levels. However, when we assayed for the protein encoded by Slc17a6, there was a reduction in protein. ${ }^{101}$ We identified miR-467b-5p, a miRNA predicted in silico to target $S l c 17 a 6$, to be differentially expressed in the same tissue, and experimentally validated this interaction using in vitro reporter assays. In this study, transcriptional output (mRNA) correlated with both promoter DNA methylation and histone modifications, following which at least one miRNA was proposed to regulate expression at a translational level, demonstrating that an early-life environmental exposure can exert complex, independent effects on gene expression. $^{101}$

Finally, it is often difficult to discern whether epigenetic marks, such as DNA methylation and post-translational histone modifications which are associated with gene expression changes following an early-life environmental exposure, are a cause or a consequence of the change in transcription. As a result, the relationship between these epigenetic modifications and gene expression is often best described as correlative in nature.

\section{Concluding remarks}

The field of $\mathrm{DOHaD}$ has historically focused on characterizing the long-term health consequences of early-life environmental exposures. There is emerging evidence that these early-life exposures can affect the epigenome, which has the potential not only to impact gene expression and phenotype but may also be stably remembered for a lifetime. The assays available to investigate the epigenome are now well within the reach of all investigators. This review covers essential concepts in epigenetics which are relevant to the $\mathrm{DOHaD}$ field, and highlights potential pitfalls as well as key considerations for study design and interpretation. We look forward to an exciting new era of $\mathrm{DOHaD}$ studies which will bring us closer to understanding not only the impact of early-life environmental exposures on health and disease in later life, but also greater knowledge of the role of epigenetics in mediating such phenomena.

\section{Acknowledgments}

The authors would like to thank James Cuffe, Vicki Clifton and Neil Youngson for their thoughtful and constructive feedback during the preparation of this manuscript. The authors apologize to the authors of relevant studies that we were unable to discuss due to space constraints.

\section{Financial Support}

L.Y. was supported by an Australian Postgraduate Award and by Mater Research-UQ (Frank Clair Scholarship). S.C. was supported by an Australian Research Council Future Fellowship (FT100100333). This work was also supported by funding from the Mater Foundation. The Translational Research Institute is supported by a grant from the Australian Government.

\section{Conflicts of Interest}

None.

\section{References}

1. Hyatt MA, Budge H, Symonds ME. Early developmental influences on hepatic organogenesis. Organogenesis. 2008; 4, 170-175.

2. Luyckx VA, Brenner BM. The clinical importance of nephron mass. J Am Soc Nephrol. 2010; 21, 898-910.

3. Waddington $\mathrm{CH}$. The pupal contraction as an epigenetic crisis in Drosophila. Proc Zool Soc Lond A. 1942; 111, 181-188.

4. Wu C, Morris JR. Genes, genetics, and epigenetics: a correspondence. Science. 2001; 293, 1103-1105.

5. National Institutes of Health. Overview of the Roadmap Epigenomics Project. National Institutes of Health, 2010. Retrieved 20 June 2016 from http://www.roadmapepigenomics. org/overview.

6. Egger G, Liang G, Aparicio A, Jones PA. Epigenetics in human disease and prospects for epigenetic therapy. Nature. 2004; 429, 457-463.

7. Godfrey KM, Costello PM, Lillycrop KA. The developmental environment, epigenetic biomarkers and long-term health. J Dev Orig Health Dis. 2015; 6, 399-406.

8. Bird A. DNA methylation patterns and epigenetic memory. Genes Dev. 2002; 16, 6-21.

9. Tomizawa S, Kobayashi H, Watanabe T, et al. Dynamic stagespecific changes in imprinted differentially methylated regions during early mammalian development and prevalence of nonCpG methylation in oocytes. Development. 2011; 138, 811-820.

10. Ziller MJ, Muller F, Liao J, et al. Genomic distribution and inter-sample variation of non-CpG methylation across human cell types. PLoS Genet. 2011; 7, e1002389.

11. Xie W, Barr CL, Kim A, et al. Base-resolution analyses of sequence and parent-of-origin dependent DNA methylation in the mouse genome. Cell. 2012; 148, 816-831.

12. Varley KE, Gertz J, Bowling KM, et al. Dynamic DNA methylation across diverse human cell lines and tissues. Genome Res. 2013; 23, 555-567.

13. Guo JU, Su Y, Shin JH, et al. Distribution, recognition and regulation of non-CpG methylation in the adult mammalian brain. Nat Neurosci. 2014; 17, 215-222.

14. Bestor TH. Activation of mammalian DNA methyltransferase by cleavage of a $\mathrm{Zn}$ binding regulatory domain. $E M B O$ J. 1992; 11, 2611-2617. 
15. Okano M, Bell DW, Haber DA, Li E. DNA methyltransferases Dnmt3a and Dnmt3b are essential for de novo methylation and mammalian development. Cell. 1999; 99, 247-257.

16. Goll MG, Bestor TH. Eukaryotic cytosine methyltransferases. Annu Rev Biochem. 2005; 74, 481-514.

17. Hata K, Okano M, Lei H, Li E. Dnmt3L cooperates with the Dnmt3 family of de novo DNA methyltransferases to establish maternal imprints in mice. Development. 2002; 129, 1983-1993.

18. Suetake I, Shinozaki F, Miyagawa J, Takeshima H, Tajima S. DNMT3L stimulates the DNA methylation activity of Dnmt3a and Dnmt3b through a direct interaction. J Biol Chem. 2004; 279, 27816-27823.

19. Gowher H, Liebert K, Hermann A, Xu G, Jeltsch A. Mechanism of stimulation of catalytic activity of Dnmt3A and Dnmt3B DNA-(cytosine-C5)-methyltransferases by Dnmt3L.J Biol Chem. 2005; 280, 13341-13348.

20. Zhu B, Zheng Y, Angliker H, et al. 5-Methylcytosine DNA glycosylase activity is also present in the human MBD4 (G/T mismatch glycosylase) and in a related avian sequence. Nucleic Acids Res. 2000; 28, 4157-4165.

21. Rai K, Huggins IJ, James SR, et al. DNA demethylation in zebrafish involves the coupling of a deaminase, a glycosylase, and Gadd45. Cell. 2008; 135, 1201-1212.

22. Metivier R, Gallais R, Tiffoche C, et al. Cyclical DNA methylation of a transcriptionally active promoter. Nature. 2008; 452, 45-50.

23. Kangaspeska S, Stride B, Metivier R, et al. Transient cyclical methylation of promoter DNA. Nature. 2008; 452, 112-115.

24. Ito S, Shen L, Dai Q, et al. Tet proteins can convert 5-methylcytosine to 5-formylcytosine and 5-carboxylcytosine. Science. 2011; 333, 1300-1303.

25. He YF, Li BZ, Li Z, et al. Tet-mediated formation of 5-carboxylcytosine and its excision by TDG in mammalian DNA. Science. 2011; 333, 1303-1307.

26. Pfaffeneder T, Hackner B, Truss M, et al. The discovery of 5-formylcytosine in embryonic stem cell DNA. Angew Chem Int Ed Engl. 2011; 50, 7008-7012.

27. Bhutani N, Burns DM, Blau HM. DNA demethylation dynamics. Cell. 2011; 146, 866-872.

28. Rougier N, Bourc'his D, Gomes DM, et al. Chromosome methylation patterns during mammalian preimplantation development. Genes Dev. 1998; 12, 2108-2113.

29. Carlson LL, Page AW, Bestor TH. Properties and localization of DNA methyltransferase in preimplantation mouse embryos: implications for genomic imprinting. Genes Dev. 1992; 6, 2536-2541.

30. Howell CY, Bestor TH, Ding F, et al. Genomic imprinting disrupted by a maternal effect mutation in the Dnmt 1 gene. Cell. 2001; 104, 829-838.

31. Esteller M. Cancer epigenomics: DNA methylomes and histonemodification maps. Nat Rev Genet. 2007; 8, 286-298.

32. Stein R, Razin A, Cedar H. In vitro methylation of the hamster adenine phosphoribosyltransferase gene inhibits its expression in mouse L cells. Proc Natl Acad Sci U S A. 1982; 79, 3418-3422.

33. Bird AP. CpG-rich islands and the function of DNA methylation. Nature. 1986; 321, 209-213.

34. Schubeler D, Lorincz MC, Cimbora DM, et al. Genomic targeting of methylated DNA: influence of methylation on transcription, replication, chromatin structure, and histone acetylation. Mol Cell Biol. 2000; 20, 9103-9112.

35. Lorincz MC, Dickerson DR, Schmitt M, Groudine M. Intragenic DNA methylation alters chromatin structure and elongation efficiency in mammalian cells. Nat Struct Mol Biol. 2004; 11, 1068-1075.

36. Gelfman S, Cohen N, Yearim A, Ast G. DNA-methylation effect on cotranscriptional splicing is dependent on GC architecture of the exon-intron structure. Genome Res. 2013; 23, 789-799.

37. Maunakea AK, Chepelev I, Cui K, Zhao K. Intragenic DNA methylation modulates alternative splicing by recruiting $\mathrm{MeCP} 2$ to promote exon recognition. Cell Res. 2013; 23, 1256-1269.

38. Tobi EW, Lumey LH, Talens RP, et al. DNA methylation differences after exposure to prenatal famine are common and timing- and sex-specific. Hum Mol Genet. 2009; 18, 4046-4053.

39. Kaminen-Ahola N, Ahola A, Maga M, et al. Maternal ethanol consumption alters the epigenotype and the phenotype of offspring in a mouse model. PLoS Genet. 2010; 6, e1000811.

40. Downing C, Johnson TE, Larson C, et al. Subtle decreases in DNA methylation and gene expression at the mouse Igf2 locus following prenatal alcohol exposure: effects of a methylsupplemented diet. Alcohol. 2011; 45, 65-71.

41. Bekdash RA, Zhang C, Sarkar DK. Gestational choline supplementation normalized fetal alcohol-induced alterations in histone modifications, DNA methylation, and proopiomelanocortin (POMC) gene expression in betaendorphin-producing POMC neurons of the hypothalamus. Alcohol Clin Exp Res. 2013; 37, 1133-1142.

42. Laufer BI, Mantha K, Kleiber ML, et al. Long-lasting alterations to DNA methylation and ncRNAs could underlie the effects of fetal alcohol exposure in mice. Dis Model Mech. 2013; 6, 977-992.

43. Kile ML, Houseman EA, Baccarelli AA, et al. Effect of prenatal arsenic exposure on DNA methylation and leukocyte subpopulations in cord blood. Epigenetics. 2014; 9, 774-782.

44. Silver MJ, Kessler NJ, Hennig BJ, et al. Independent genomewide screens identify the tumor suppressor VTRNA2-1 as a human epiallele responsive to periconceptional environment. Genome Biol. 2015; 16, 118.

45. Tobi EW, Goeman JJ, Monajemi R, et al. DNA methylation signatures link prenatal famine exposure to growth and metabolism. Nat Commun. 2014; 5, 5592.

46. Amarasekera M, Martino D, Ashley S, et al. Genome-wide DNA methylation profiling identifies a folate-sensitive region of differential methylation upstream of ZFP57-imprinting regulator in humans. FASEB J. 2014; 28, 4068-4076.

47. Thompson RF, Fazzari MJ, Niu H, et al. Experimental intrauterine growth restriction induces alterations in DNA methylation and gene expression in pancreatic islets of rats. J Biol Chem. 2010; 285, 15111-15118.

48. Kovacheva VP, Mellott TJ, Davison JM, et al. Gestational choline deficiency causes global and Igf2 gene DNA hypermethylation by up-regulation of Dnmt1 expression. J Biol Chem. 2007; 282, 31777-31788.

49. Gardebjer EM, Anderson ST, Pantaleon M, Wlodek ME, Moritz KM. Maternal alcohol intake around the time of conception causes glucose intolerance and insulin insensitivity in rat offspring, which is exacerbated by a postnatal high-fat diet. FASEB J. 2015; 29, 2690-2701. 
50. Stover PJ. One-carbon metabolism-genome interactions in folateassociated pathologies. J Nutr. 2009; 139, 2402-2405.

51. Ngai YF, Sulistyoningrum DC, O’Neill R, et al. Prenatal alcohol exposure alters methyl metabolism and programs serotonin transporter and glucocorticoid receptor expression in brain. Am J Physiol Regul Integr Comp Physiol. 2015; 309, R613-R622.

52. Drake AJ, O'Shaughnessy PJ, Bhattacharya S, et al. In utero exposure to cigarette chemicals induces sex-specific disruption of one-carbon metabolism and DNA methylation in the human fetal liver. BMC Med. 2015; 13, 18.

53. Garro AJ, McBeth DL, Lima V, Lieber CS. Ethanol consumption inhibits fetal DNA methylation in mice: implications for the fetal alcohol syndrome. Alcohol Clin Exp Res. 1991; 15, 395-398.

54. Haycock PC. Fetal alcohol spectrum disorders: the epigenetic perspective. Biol Reprod. 2009; 81, 607-617.

55. McKay JA, Waltham KJ, Williams EA, Mathers JC. Folate depletion during pregnancy and lactation reduces genomic DNA methylation in murine adult offspring. Genes Nutr. 2011; 6, 189-196.

56. Breton CV, Siegmund KD, Joubert BR, et al. Prenatal tobacco smoke exposure is associated with childhood DNA CpG methylation. PLoS One. 2014; 9, e99716.

57. Cheung P, Allis CD, Sassone-Corsi P. Signaling to chromatin through histone modifications. Cell. 2000; 103, 263-271.

58. Luger K, Mader AW, Richmond RK, Sargent DF, Richmond TJ. Crystal structure of the nucleosome core particle at $2.8 \mathrm{~A}$ resolution. Nature. 1997; 389, 251-260.

59. Lanzuolo C, Orlando V. The function of the epigenome in cell reprogramming. Cell Mol Life Sci. 2007; 64, 1043-1062.

60. Paulson JR, Taylor SS. Phosphorylation of histones 1 and 3 and nonhistone high mobility group 14 by an endogenous kinase in HeLa metaphase chromosomes. J Biol Chem. 1982; 257, 6064-6072.

61. Davie JR, Murphy LC. Level of ubiquitinated histone H2B in chromatin is coupled to ongoing transcription. Biochemistry. 1990; 29, 4752-4757.

62. Wondrak GT, Cervantes-Laurean D, Jacobson EL, Jacobson MK. Histone carbonylation in vivo and in vitro. Biochem J. 2000; 351(Pt 3), 769-777.

63. Poirier GG, de Murcia G, Jongstra-Bilen J, Niedergang C, Mandel P. Poly(ADP-ribosyl)ation of polynucleosomes causes relaxation of chromatin structure. Proc Natl Acad Sci US A. 1982; 79, 3423-3427.

64. Shiio Y, Eisenman RN. Histone sumoylation is associated with transcriptional repression. Proc Natl Acad Sci U S A. 2003; 100, 13225-13230.

65. Bannister AJ, Kouzarides T. Regulation of chromatin by histone modifications. Cell Res. 2011; 21, 381-395.

66. Shi X, Hong T, Walter KL, et al. ING2 PHD domain links histone $\mathrm{H} 3$ lysine 4 methylation to active gene repression. Nature. 2006; 442, 96-99.

67. Hassan AH, Prochasson P, Neely KE, et al. Function and selectivity of bromodomains in anchoring chromatin-modifying complexes to promoter nucleosomes. Cell. 2002; 111, 369-379.

68. Strahl BD, Allis CD. The language of covalent histone modifications. Nature. 2000; 403, 41-45.

69. Vermeulen M, Mulder KW, Denissov S, et al. Selective anchoring of TFIID to nucleosomes by trimethylation of histone $\mathrm{H} 3$ lysine 4. Cell. 2007; 131, 58-69.
70. Cao R, Wang L, Wang H, et al. Role of histone H3 lysine 27 methylation in Polycomb-group silencing. Science. 2002; 298, 1039-1043.

71. Bernstein BE, Mikkelsen TS, Xie X, et al. A bivalent chromatin structure marks key developmental genes in embryonic stem cells. Cell. 2006; 125, 315-326.

72. Stevens A, Begum G, Cook A, et al. Epigenetic changes in the hypothalamic proopiomelanocortin and glucocorticoid receptor genes in the ovine fetus after periconceptional undernutrition. Endocrinology. 2010; 151, 3652-3664.

73. Wang X, Gomutputra P, Wolgemuth DJ, Baxi LV. Acute alcohol exposure induces apoptosis and increases histone $\mathrm{H} 3 \mathrm{~K} 9 / 18$ acetylation in the mid-gestation mouse lung. Reprod Sci. 2010; 17, 384-390.

74. Zinkhan EK, Fu Q, Wang Y, et al. Maternal hyperglycemia disrupts histone 3 lysine 36 trimethylation of the IGF-1 gene. J Nutr Metab. 2012; 2012, 930364.

75. Davison JM, Mellott TJ, Kovacheva VP, Blusztajn JK. Gestational choline supply regulates methylation of histone $\mathrm{H} 3$, expression of histone methyltransferases G9a (Kmtlc) and Suv39h1 (Kmtla), and DNA methylation of their genes in rat fetal liver and brain. J Biol Chem. 2009; 284, 1982-1989.

76. Mehedint MG, Niculescu MD, Craciunescu CN, Zeisel SH. Choline deficiency alters global histone methylation and epigenetic marking at the Re 1 site of the calbindin 1 gene. FASEB J. 2010; 24, 184-195.

77. Suter MA, Abramovici AR, Griffin E, et al. In utero nicotine exposure epigenetically alters fetal chromatin structure and differentially regulates transcription of the glucocorticoid receptor in a rat model. Birth Defects Res A Clin Mol Teratol. 2015; 103, 583-588.

78. Tyler CR, Weber JA, Labrecque M, et al. ChIP-Seq analysis of the adult male mouse brain after developmental exposure to arsenic. Data Brief. 2015; 5, 248-254.

79. Yang KF, Cai W, Xu JL, Shi W. Maternal high-fat diet programs Wnt genes through histone modification in the liver of neonatal rats. J Mol Endocrinol. 2012; 49, $107-114$

80. Wang KC, Chang HY. Molecular mechanisms of long noncoding RNAs. Mol Cell. 2011; 43, 904-914.

81. Nagano T, Mitchell JA, Sanz LA, et al. The air noncoding RNA epigenetically silences transcription by targeting G9a to chromatin. Science. 2008; 322, 1717-1720.

82. Bonasio R, Tu S, Reinberg D. Molecular signals of epigenetic states. Science. 2010; 330, 612-616.

83. Kim VN, Han J, Siomi MC. Biogenesis of small RNAs in animals. Nat Rev Mol Cell Biol. 2009; 10, 126-139.

84. Iwasaki YW, Siomi MC, Siomi H. PIWI-interacting RNA: its biogenesis and functions. Annu Rev Biochem. 2015; 84, 405-433.

85. Landgraf P, Rusu M, Sheridan R, et al. A mammalian microRNA expression atlas based on small RNA library sequencing. Cell. 2007; 129, 1401-1414.

86. Huang Y, Zhang JL, Yu XL, et al. Molecular functions of small regulatory noncoding RNA. Biochemistry (Mosc). 2013; 78, 221-230.

87. Bartel DP. MicroRNAs: genomics, biogenesis, mechanism, and function. Cell. 2004; 116, 281-297.

88. Yekta S, Shih IH, Bartel DP. MicroRNA-directed cleavage of HOXB8 mRNA. Science. 2004; 304, 594-596. 
89. Bagga S, Bracht J, Hunter S, et al. Regulation by let-7 and lin-4 miRNAs results in target mRNA degradation. Cell. 2005; 122, 553-563.

90. Olsen PH, Ambros V. The lin-4 regulatory RNA controls developmental timing in Caenorhabditis elegans by blocking LIN-14 protein synthesis after the initiation of translation. Dev Biol. 1999; 216, 671-680.

91. Pillai RS, Bhattacharyya SN, Artus CG, et al. Inhibition of translational initiation by let-7 microRNA in human cells. Science. 2005; 309, 1573-1576.

92. Djuranovic S, Nahvi A, Green R. miRNA-mediated gene silencing by translational repression followed by mRNA deadenylation and decay. Science. 2012; 336, 237-240.

93. Saraiya AA, Li W, Wang CC. Transition of a microRNA from repressing to activating translation depending on the extent of base pairing with the target. PLoS One. 2013; 8, e55672.

94. Vasudevan S, Tong Y, Steitz JA. Switching from repression to activation: microRNAs can up-regulate translation. Science. 2007; 318, 1931-1934.

95. Lewis BP, Burge CB, Bartel DP. Conserved seed pairing, often flanked by adenosines, indicates that thousands of human genes are microRNA targets. Cell. 2005; 120, 15-20.

96. Xie X, Lu J, Kulbokas EJ, et al. Systematic discovery of regulatory motifs in human promoters and 3' UTRs by comparison of several mammals. Nature. 2005; 434, 338-345.

97. Brennecke J, Stark A, Russell RB, Cohen SM. Principles of microRNA-target recognition. PLoS Biol. 2005; 3, e85.

98. Krek A, Grun D, Poy MN, et al. Combinatorial microRNA target predictions. Nat Genet. 2005; 37, 495-500.

99. Wang LL, Zhang Z, Li Q, et al. Ethanol exposure induces differential microRNA and target gene expression and teratogenic effects which can be suppressed by folic acid supplementation. Hum Reprod. 2009; 24, 562-579.

100. Soares AR, Pereira PM, Ferreira V, et al. Ethanol exposure induces upregulation of specific microRNAs in zebrafish embryos. Toxicol Sci. 2012; 127, 18-28.

101. Zhang CR, Ho MF, Vega MC, Burne TH, Chong S. Prenatal ethanol exposure alters adult hippocampal VGLUT2 expression with concomitant changes in promoter DNA methylation, $\mathrm{H} 3 \mathrm{~K} 4$ trimethylation and miR-467b-5p levels. Epigenetics Chromatin. 2015; 8, 40.

102. Maccani MA, Marsit CJ. Exposure and fetal growth-associated miRNA alterations in the human placenta. Clin Epigenetics. 2011; 2, 401-404.

103. Balaraman S, Lunde ER, Sawant O, et al. Maternal and neonatal plasma microRNA biomarkers for fetal alcohol exposure in an ovine model. Alcohol Clin Exp Res. 2014; 38, 1390-1400.

104. Vrijens K, Bollati V, Nawrot TS. MicroRNAs as potential signatures of environmental exposure or effect: a systematic review. Environ Health Perspect. 2015; 123, 399-411.

105. Zhang Y, Fatima N, Dufau ML. Coordinated changes in DNA methylation and histone modifications regulate silencing/ derepression of luteinizing hormone receptor gene transcription. Mol Cell Biol. 2005; 25, 7929-7939.

106. Nan X, Ng HH, Johnson CA, et al. Transcriptional repression by the methyl-CpG-binding protein $\mathrm{MeCP} 2$ involves a histone deacetylase complex. Nature. 1998; 393, 386-389.
107. Jones PL, Veenstra GJ, Wade PA, et al. Methylated DNA and $\mathrm{MeCP} 2$ recruit histone deacetylase to repress transcription. Nat Genet. 1998; 19, 187-191.

108. Eden S, Hashimshony T, Keshet I, Cedar H, Thorne AW. DNA methylation models histone acetylation. Nature. 1998; 394, 842 .

109. Zhao Q, Rank G, Tan YT, et al. PRMT5-mediated methylation of histone H4R3 recruits DNMT3A, coupling histone and DNA methylation in gene silencing. Nat Struct Mol Biol. 2009; 16, 304-311.

110. Stewart KR, Veselovska L, Kim J, et al. Dynamic changes in histone modifications precede de novo DNA methylation in oocytes. Genes Dev. 2015; 29, 2449-2462.

111. Vire E, Brenner C, Deplus R, et al. The Polycomb group protein EZH2 directly controls DNA methylation. Nature. 2006; 439, 871-874.

112. Ooi SK, Qiu C, Bernstein E, et al. DNMT3L connects unmethylated lysine 4 of histone $\mathrm{H} 3$ to de novo methylation of DNA. Nature. 2007; 448, 714-717.

113. Cedar H, Bergman Y. Linking DNA methylation and histone modification: patterns and paradigms. Nat Rev Genet. 2009; 10, 295-304.

114. Rose NR, Klose RJ. Understanding the relationship between DNA methylation and histone lysine methylation. Biochim Biophys Acta. 2014; 1839, 1362-1372.

115. Du J, Johnson LM, Jacobsen SE, Patel DJ. DNA methylation pathways and their crosstalk with histone methylation. Nat Rev Mol Cell Biol. 2015; 16, 519-532.

116. Han L, Witmer PD, Casey E, Valle D, Sukumar S. DNA methylation regulates microRNA expression. Cancer Biol Ther. 2007; 6, 1284-1288.

117. Sun BK, Deaton AM, Lee JT. A transient heterochromatic state in Xist preempts X inactivation choice without RNA stabilization. Mol Cell. 2006; 21, 617-628.

118. Schmitz KM, Mayer C, Postepska A, Grummt I. Interaction of noncoding RNA with the rDNA promoter mediates recruitment of DNMT3b and silencing of rRNA genes. Genes Dev. 2010; 24, 2264-2269.

119. Tomikawa J, Shimokawa H, Uesaka M, et al. Single-stranded noncoding RNAs mediate local epigenetic alterations at gene promoters in rat cell lines. J Biol Chem. 2011; 286, 34788-34799.

120. Holz-Schietinger C, Reich NO. RNA modulation of the human DNA methyltransferase 3A. Nucleic Acids Res. 2012; 40, 8550-8557.

121. Di Ruscio A, Ebralidze AK, Benoukraf T, et al. DNMT1interacting RNAs block gene-specific DNA methylation. Nature. 2013; 503, 371-376.

122. Zhou J, Yang L, Zhong T, et al. H19 lncRNA alters DNA methylation genome wide by regulating S-adenosylhomocysteine hydrolase. Nat Commun. 2015; 6, 10221.

123. Watanabe T, Tomizawa S, Mitsuya K, et al. Role for piRNAs and noncoding RNA in de novo DNA methylation of the imprinted mouse Rasgrf1 locus. Science. 2011; 332, $848-852$.

124. Kuramochi-Miyagawa S, Watanabe T, Gotoh K, et al. DNA methylation of retrotransposon genes is regulated by Piwi family members MILI and MIWI2 in murine fetal testes. Genes Dev. 2008; 22, 908-917. 
125. Braconi C, Huang N, Patel T. MicroRNA-dependent regulation of DNA methyltransferase-1 and tumor suppressor gene expression by interleukin- 6 in human malignant cholangiocytes. Hepatology. 2010; 51, 881-890.

126. Huang J, Wang Y, Guo Y, Sun S. Down-regulated microRNA-152 induces aberrant DNA methylation in hepatitis $B$ virus-related hepatocellular carcinoma by targeting DNA methyltransferase 1. Hepatology. 2010; 52, 60-70.

127. Barski A, Jothi R, Cuddapah S, et al. Chromatin poises miRNAand protein-coding genes for expression. Genome Res. 2009; 19, 1742-1751.

128. Wu SC, Kallin EM, Zhang Y. Role of H3K27 methylation in the regulation of IncRNA expression. Cell Res. 2010; 20, 1109-1116.

129. Guil S, Esteller M. DNA methylomes, histone codes and miRNAs: tying it all together. Int J Biochem Cell Biol. 2009; 41, 87-95.

130. Peschansky VJ, Wahlestedt C. Non-coding RNAs as direct and indirect modulators of epigenetic regulation. Epigenetics. 2014; 9, 3-12.

131. Rinn JL, Kertesz M, Wang JK, et al. Functional demarcation of active and silent chromatin domains in human HOX loci by noncoding RNAs. Cell. 2007; 129, 1311-1323.

132. Tsai MC, Manor O, Wan Y, et al. Long noncoding RNA as modular scaffold of histone modification complexes. Science. 2010; 329, 689-693.

133. Noonan EJ, Place RF, Pookot D, et al. miR-449a targets HDAC-1 and induces growth arrest in prostate cancer. Oncogene. 2009; 28, 1714-1724.

134. Wong CF, Tellam RL. MicroRNA-26a targets the histone methyltransferase Enhancer of Zeste homolog 2 during myogenesis. J Biol Chem. 2008; 283, 9836-9843.

135. Messerschmidt DM, Knowles BB, Solter D. DNA methylation dynamics during epigenetic reprogramming in the germline and preimplantation embryos. Genes Dev. 2014; 28, 812-828.

136. Li E. Chromatin modification and epigenetic reprogramming in mammalian development. Nat Rev Genet. 2002; 3, 662-673.

137. Santos F, Dean W. Epigenetic reprogramming during early development in mammals. Reproduction. 2004; 127, 643-651.

138. Morgan HD, Santos F, Green K, Dean W, Reik W. Epigenetic reprogramming in mammals. Hum Mol Genet. 2005; 14(Spec No 1), R47-R58.

139. Reik W, Dean W, Walter J. Epigenetic reprogramming in mammalian development. Science. 2001; 293, 1089-1093.

140. Oswald J, Engemann S, Lane N, et al. Active demethylation of the paternal genome in the mouse zygote. Curr Biol. 2000; 10, 475-478.

141. Monk M, Boubelik M, Lehnert S. Temporal and regional changes in DNA methylation in the embryonic, extraembryonic and germ cell lineages during mouse embryo development. Development. 1987; 99, 371-382.

142. Bhutani N, Brady JJ, Damian M, et al. Reprogramming towards pluripotency requires AID-dependent DNA demethylation. Nature. 2010; 463, 1042-1047.

143. Liu Y, Balaraman Y, Wang G, Nephew KP, Zhou FC. Alcohol exposure alters DNA methylation profiles in mouse embryos at early neurulation. Epigenetics. 2009; 4, 500-511.

144. Chen PY, Ganguly A, Rubbi L, et al. Intrauterine calorie restriction affects placental DNA methylation and gene expression. Physiol Genomics. 2013; 45, 565-576.
145. Ivanova E, Chen JH, Segonds-Pichon A, Ozanne SE, Kelsey G. DNA methylation at differentially methylated regions of imprinted genes is resistant to developmental programming by maternal nutrition. Epigenetics. 2012; 7, 1200-1210.

146. Radford EJ, Isganaitis E, Jimenez-Chillaron J, et al. An unbiased assessment of the role of imprinted genes in an intergenerational model of developmental programming. PLoS Genet. 2012; 8, e1002605.

147. Lecomte V, Youngson NA, Maloney CA, Morris MJ. Parental programming: how can we improve study design to discern the molecular mechanisms? Bioessays. 2013; 35, 787-793.

148. Kawasaki Y, Lee J, Matsuzawa A, et al. Active DNA demethylation is required for complete imprint erasure in primordial germ cells. Sci Rep. 2014; 4, 3658.

149. Sasaki H, Matsui Y. Epigenetic events in mammalian germ-cell development: reprogramming and beyond. Nat Rev Genet. 2008; 9, 129-140.

150. Lucifero D, Mann MR, Bartolomei MS, Trasler JM. Gene-specific timing and epigenetic memory in oocyte imprinting. Hum Mol Genet. 2004; 13, 839-849.

151. Geuns E, De Rycke M, Van Steirteghem A, Liebaers I. Methylation imprints of the imprint control region of the SNRPN-gene in human gametes and preimplantation embryos. Hum Mol Genet. 2003; 12, 2873-2879.

152. Okae $\mathrm{H}$, Chiba $\mathrm{H}$, Hiura $\mathrm{H}$, et al. Genome-wide analysis of DNA methylation dynamics during early human development. PLoS Genet. 2014; 10, e1004868.

153. Heijmans BT, Kremer D, Tobi EW, Boomsma DI, Slagboom PE. Heritable rather than age-related environmental and stochastic factors dominate variation in DNA methylation of the human IGF2/H19 locus. Hum Mol Genet. 2007; 16, 547-554.

154. Schilling E, El Chartouni C, Rehli M. Allele-specific DNA methylation in mouse strains is mainly determined by cis-acting sequences. Genome Res. 2009; 19, 2028-2035.

155. Zhi D, Aslibekyan S, Irvin MR, et al. SNPs located at CpG sites modulate genome-epigenome interaction. Epigenetics. 2013; 8, 802-806.

156. Banovich NE, Lan X, McVicker G, et al. Methylation QTLs are associated with coordinated changes in transcription factor binding, histone modifications, and gene expression levels. PLoS Genet. 2014; 10, e1004663.

157. McClay JL, Shabalin AA, Dozmorov MG, et al. High density methylation QTL analysis in human blood via next-generation sequencing of the methylated genomic DNA fraction. Genome Biol. 2015; 16, 291.

158. Frosst $\mathrm{P}, \mathrm{Blom} \mathrm{HJ}$, Milos $\mathrm{R}$, et al. A candidate genetic risk factor for vascular disease: a common mutation in methylenetetrahydrofolate reductase. Nat Genet. 1995; 10, 111-113.

159. Stern LL, Mason JB, Selhub J, Choi SW. Genomic DNA hypomethylation, a characteristic of most cancers, is present in peripheral leukocytes of individuals who are homozygous for the C677T polymorphism in the methylenetetrahydrofolate reductase gene. Cancer Epidemiol Biomarkers Prev. 2000; 9, 849-853.

160. Friso S, Choi SW, Girelli D, et al. A common mutation in the 5,10-methylenetetrahydrofolate reductase gene affects genomic DNA methylation through an interaction with folate status. Proc Natl Acad Sci U S A. 2002; 99, 5606-5611. 
161. El-Maarri O, Kareta MS, Mikeska T, et al. A systematic search for DNA methyltransferase polymorphisms reveals a rare DNMT3L variant associated with subtelomeric hypomethylation. Hum Mol Genet. 2009; 18, 1755-1768.

162. Kadota M, Yang HH, Hu N, et al. Allele-specific chromatin immunoprecipitation studies show genetic influence on chromatin state in human genome. PLoS Genet. 2007; 3, e81.

163. Kerkel K, Spadola A, Yuan E, et al. Genomic surveys by methylation-sensitive SNP analysis identify sequence-dependent allele-specific DNA methylation. Nat Genet. 2008; 40, 904-908.

164. Haycock PC, Ramsay M. Exposure of mouse embryos to ethanol during preimplantation development: effect on DNA methylation in the H19 imprinting control region. Biol Reprod. 2009; 81, 618-627.

165. Zhang D, Cheng L, Badner JA, et al. Genetic control of individual differences in gene-specific methylation in human brain. Am J Hum Genet. 2010; 86, 411-419.

166. Oey H, Isbel L, Hickey P, Ebaid B, Whitelaw E. Genetic and epigenetic variation among inbred mouse littermates: identification of inter-individual differentially methylated regions. Epigenetics Chromatin. 2015; 8, 54.

167. Shea JM, Serra RW, Carone BR, et al. Genetic and epigenetic variation, but not diet, shape the sperm methylome. Dev Cell. 2015; 35, 750-758.

168. Ohgane J, Aikawa J, Ogura A, et al. Analysis of CpG islands of trophoblast giant cells by restriction landmark genomic scanning. Dev Genet. 1998; 22, 132-140.

169. Shiota K, Kogo Y, Ohgane J, et al. Epigenetic marks by DNA methylation specific to stem, germ and somatic cells in mice. Genes Cells. 2002; 7, 961-969.

170. Yagi S, Hirabayashi K, Sato S, et al. DNA methylation profile of tissue-dependent and differentially methylated regions (T-DMRs) in mouse promoter regions demonstrating tissuespecific gene expression. Genome Res. 2008; 18, 1969-1978.

171. Davies MN, Volta M, Pidsley R, et al. Functional annotation of the human brain methylome identifies tissue-specific epigenetic variation across brain and blood. Genome Biol. 2012; 13, R43.

172. Roadmap Epigenomics C, Kundaje A, Meuleman W, et al. Integrative analysis of 111 reference human epigenomes. Nature. 2015; 518, 317-330.

173. Leung D, Jung I, Rajagopal N, et al. Integrative analysis of haplotype-resolved epigenomes across human tissues. Nature. 2015; 518, 350-354.

174. Flanagan JM, Popendikyte V, Pozdniakovaite N, et al. Intraand interindividual epigenetic variation in human germ cells. Am J Hum Genet. 2006; 79, 67-84.

175. Farlik M, Sheffield NC, Nuzzo A, et al. Single-cell DNA methylome sequencing and bioinformatic inference of epigenomic cell-state dynamics. Cell Rep. 2015; 10, 1386-1397.

176. Novakovic B, Ryan J, Pereira N, et al. Postnatal stability, tissue, and time specific effects of AHRR methylation change in response to maternal smoking in pregnancy. Epigenetics. 2014; 9, 377-386.

177. Basil P, Li Q, Dempster EL, et al. Prenatal maternal immune activation causes epigenetic differences in adolescent mouse brain. Transl Psychiatry. 2014; 4, e434.

178. Kundakovic M, Gudsnuk K, Franks B, et al. Sex-specific epigenetic disruption and behavioral changes following low-dose in utero bisphenol A exposure. Proc Natl Acad Sci U S A. 2013; 110, 9956-9961.

179. Begum G, Davies A, Stevens A, et al. Maternal undernutrition programs tissue-specific epigenetic changes in the glucocorticoid receptor in adult offspring. Endocrinology. 2013; 154, 4560-4569.

180. El Hajj N, Pliushch G, Schneider E, et al. Metabolic programming of MEST DNA methylation by intrauterine exposure to gestational diabetes mellitus. Diabetes. 2013; 62, 1320-1328.

181. Cardenas A, Koestler DC, Houseman EA, et al. Differential DNA methylation in umbilical cord blood of infants exposed to mercury and arsenic in utero. Epigenetics. 2015; 10, 508-515.

182. Jacoby M, Gohrbandt S, Clausse V, Brons NH, Muller CP. Interindividual variability and co-regulation of DNA methylation differ among blood cell populations. Epigenetics. 2012; 7, 1421-1434.

183. Reinius LE, Acevedo N, Joerink M, et al. Differential DNA methylation in purified human blood cells: implications for cell lineage and studies on disease susceptibility. PLoS One. 2012; 7, e41361.

184. Jaffe AE, Irizarry RA. Accounting for cellular heterogeneity is critical in epigenome-wide association studies. Genome Biol. 2014; 15, R31.

185. Bauer M, Linsel G, Fink B, et al. A varying T cell subtype explains apparent tobacco smoking induced single $\mathrm{CpG}$ hypomethylation in whole blood. Clin Epigenetics. 2015; 7, 81.

186. Houseman EA, Accomando WP, Koestler DC, et al. DNA methylation arrays as surrogate measures of cell mixture distribution. BMC Bioinformatics. 2012; 13, 86.

187. Guintivano J, Aryee MJ, Kaminsky ZA. A cell epigenotype specific model for the correction of brain cellular heterogeneity bias and its application to age, brain region and major depression. Epigenetics. 2013; 8, 290-302.

188. Koestler DC, Christensen B, Karagas MR, et al. Blood-based profiles of DNA methylation predict the underlying distribution of cell types: a validation analysis. Epigenetics. 2013; 8, 816-826.

189. Langevin SM, Houseman EA, Accomando WP, et al. Leukocyte-adjusted epigenome-wide association studies of blood from solid tumor patients. Epigenetics. 2014; 9, 884-895.

190. Yousefi P, Huen K, Quach H, et al. Estimation of blood cellular heterogeneity in newborns and children for epigenome-wide association studies. Environ Mol Mutagen. 2015; 56, 751-758.

191. Singhal RP, Mays-Hoopes LL, Eichhorn GL. DNA methylation in aging of mice. Mech Ageing Dev. 1987; 41, 199-210.

192. Fraga MF, Ballestar E, Paz MF, et al. Epigenetic differences arise during the lifetime of monozygotic twins. Proc Natl Acad Sci US A. 2005; 102, 10604-10609.

193. Levesque ML, Casey KF, Szyf M, et al. Genome-wide DNA methylation variability in adolescent monozygotic twins followed since birth. Epigenetics. 2014; 9, 1410-1421.

194. Talens RP, Boomsma DI, Tobi EW, et al. Variation, patterns, and temporal stability of DNA methylation: considerations for epigenetic epidemiology. FASEB J. 2010; 24, 3135-3144.

195. Eckhardt F, Lewin J, Cortese R, et al. DNA methylation profiling of human chromosomes 6, 20 and 22. Nat Genet. 2006; 38, 1378-1385. 
196. Carone BR, Fauquier L, Habib N, et al. Paternally induced transgenerational environmental reprogramming of metabolic gene expression in mammals. Cell. 2010; 143, 1084-1096.

197. Volkmar M, Dedeurwaerder S, Cunha DA, et al. DNA methylation profiling identifies epigenetic dysregulation in pancreatic islets from type 2 diabetic patients. EMBO J. 2012; 31, 1405-1426.

198. Chen G, Gharib TG, Huang CC, et al. Discordant protein and mRNA expression in lung adenocarcinomas. Mol Cell

Proteomics. 2002; 1, 304-313.
199. Ghazalpour A, Bennett B, Petyuk VA, et al. Comparative analysis of proteome and transcriptome variation in mouse. PLoS Genet. 2011; 7, e1001393.

200. Lie S, Morrison JL, Williams-Wyss O, et al. Impact of embryo number and maternal undernutrition around the time of conception on insulin signaling and gluconeogenic factors and microRNAs in the liver of fetal sheep. Am J Physiol Endocrinol Metab. 2014; 306, E1013-E1024. 